University of Nebraska - Lincoln

DigitalCommons@University of Nebraska - Lincoln

9-2009

\title{
Chemically decorated boron-nitride nanoribbons
}

\author{
Xiao-jun Wu \\ University of Nebraska-Lincoln, xwu4@unl.edu \\ Men-hao Wu \\ University of Nebraska-Lincoln \\ Xiao Cheng Zeng \\ University of Nebraska-Lincoln, xzeng1@unl.edu
}

Follow this and additional works at: https://digitalcommons.unl.edu/chemzeng

Part of the Chemistry Commons

Wu, Xiao-jun; Wu, Men-hao; and Zeng, Xiao Cheng, "Chemically decorated boron-nitride nanoribbons" (2009). Xiao Cheng Zeng Publications. 101.

https://digitalcommons.unl.edu/chemzeng/101

This Article is brought to you for free and open access by the Published Research - Department of Chemistry at DigitalCommons@University of Nebraska - Lincoln. It has been accepted for inclusion in Xiao Cheng Zeng Publications by an authorized administrator of DigitalCommons@University of Nebraska - Lincoln. 


\title{
Chemically decorated boron-nitride nanoribbons
}

\author{
Xiao-jun Wu, Men-hao Wu, and Xiao Cheng Zeng \\ Department of Chemistry and Nebraska Center for Materials and Nanoscience, \\ University of Nebraska-Lincoln, Lincoln, NB 68588, USA \\ Corresponding author - Xiao Cheng Zeng, email xzeng1@unl.edu
}

\begin{abstract}
Motivated by recent studies of graphenen nanoribbons (GNRs), we explored electronic properties of pure and chemically modified boron nitride nanoribbons (BNNRs) using the density functional theory method. Pure BNNRs with both edges fully saturated by hydrogen are semiconducting with wide band gaps. Values of the band gap depend on the width and the type of edge. The chemical decoration of BNNRs' edges with four different functional groups, including $-\mathrm{F},-\mathrm{Cl},-\mathrm{OH}$, and $-\mathrm{NO}_{2}$, was investigated. The band-gap modulation by chemical decoration may be exploited for nanoelectronic applications.
\end{abstract}

Keywords: boron-nitride nanoribbons, chemical modification

Graphene, a single layer of graphite, has attracted considerable research attention recently due to its intriguing physical properties and potential applications in nanoelectronics [1-5]. Both experimental and theoretical studies have shown that, by carving a graphene sheet into one-dimensional nanoribbons, the electronic band gap of the graphene nanoribbon (GNR) opens up and is dependent on its width and crystallographic orientation [6-16]. These findings render graphene-based band-structure engineering conceivable [14-21]. Moreover, theoretical calculations have shown that half-metallicity may be realized in GNR either by applying an external in-plane electric field or by chemically functionalizing zigzag-edges of GNR with different groups such as $\mathrm{H}, \mathrm{COOH}, \mathrm{OH}, \mathrm{NO}_{2}, \mathrm{NH}_{3}, \mathrm{CH}_{3}$, etc. [2326]. Half-metallic GNR may find application in spintronics.

Two-dimensional hexagonal boron nitride (h-BN) is structurally similar to graphene, and has been fabricated experimentally [27]. Unlike graphene, the h-BN has a large energy band gap due to strong ionicity of BN. Hence, it is expected that nanoribbons carved out of h-BN also have large band gaps. It has been shown that the band gap of boron nitride nanoribbons (BNNRs) whose zigzag edges are passivated by hydrogen decreases with increasing width, while that of BNNRs with armchair edges oscillate periodically with the width [28-34]. Moreover, recent calculations indicate that electronic properties of BNNRs can be modulated by applying external transversal electric field, and BNNRs may undergo metallic to semiconducting to half-metallic transition [29-33]. The half-metallicity is also found in BNNRs with only one edge passivated with hydrogen [34]. These findings suggest potential applications of BNNRs in nanoelectronic devices. In this work, we show, through density functional theory (DFT) calculations, that electronic properties of BNNRs can be modulated by chemical decoration at the ribbons' edges. Four chemical functional groups were considered, including $-\mathrm{H},-\mathrm{NO}_{2},-\mathrm{F}$, and $-\mathrm{Cl}$. Our studies suggest that edge decoration is a viable way to tailor electronic properties of BNNRs.

The first-principles calculations were based on the linear combination of atomic orbital DFT method implemented in DMol3 package* $[35,36]$. The generalized gradient approximation (GGA) in the Perdew-Burke-Ernzerhof (PBE) form as well as an all-electron double numerical basis set with polarized function (DNP) were chosen for the spin-unrestricted DFT calculation [37]. The real-space global cutoff radius was set to be $3.70 \AA$. To simulate chemically modified BNNRs, a tetragonal supercell was selected. BNNRs with either zigzag or armchair edges were considered for pristine models. The nearest distance between two neighboring BNNRs is greater than $30 \AA$. The $k$-points sampling was employed using the Monkhorst-Pack scheme with spacing of $0.04 \AA^{-1}$ [38]. The structures were fully optimized with no constrain, and the forces on atoms were less than $0.05 \mathrm{eV} \cdot \AA^{-1}$ after geometric optimization.

* DMol3 is a density functional theory quantum mechanical package available from Accelrys. Delley Software, Inc. 
We first computed the electronic properties of pure BNNRs with zigzag and armchair edges, respectively, all passivated with hydrogen atoms, as shown in Figures 1 and 2. The width of BNNR $w$ is defined as the number of rings along the direction normal to the ribbon axis. For BNNR with zigzag edges, the optimized periodic length of the ribbon is $2.514 \AA$. The average B-N bond length is $1.454 \AA$ for $w=4$, the same as that within a BN sheet (1.454 A with the same level of calculation). The $\mathrm{N}-\mathrm{H}$ and $\mathrm{B}-\mathrm{H}$ bond lengths are 1.016 and $1.202 \AA$, respectively. The Hirshfeld charge analysis indicates that average charge transfer from $B$ to $\mathrm{N}$ is about $0.19 e$ inside the ribbon. At the edges, average charge transfer from $\mathrm{H}$ to $\mathrm{N}$ is about $0.12 e$, and $-0.052 e$ from $\mathrm{H}$ to $\mathrm{B}$. The latter charge is too small to be of qualitative significance. For BNNR with armchair edges, the optimized periodic length along the ribbon direction is $4.382 \AA$. The average $\mathrm{B}-\mathrm{N}$ bond length is $1.452 \AA$ for $w=4$, and the $\mathrm{N}-\mathrm{H}$ and $\mathrm{B}-\mathrm{H}$ bond lengths are 1.016 and $1.209 \AA$, respectively, similar to those in BNNR with zigzag edges. The charge analysis indicates that the charge transfer from $B$ to $\mathrm{N}$ is about $0.19 e$ inside the ribbon, about $0.11 e$ from $\mathrm{H}$ to $\mathrm{N}$, and $-0.056 e$ from $\mathrm{B}$ to $\mathrm{H}$ at the edges. Clearly, the edge shape of BNNRs does not affect the structures and charge transfer behavior inside the BNNR.
In Figures 1 and 2, electronic band structures and energy band gaps of BNNR with zigzag or armchair edges are presented. Pure BNNRs with zigzag edges are semiconducting with indirect band gaps $>4.0 \mathrm{eV}$, so are those with armchair edges but with direct band gaps $>4.5 \mathrm{eV}$. Near the Fermi level, profiles of electronic structures depend on BNNR's edge structure, but are independent of their width $w$. The band gap of BNNRs with zigzag edges decreases monotonically with increasing $w$, while those with armchair edges oscillates periodically with a period of $w=1.5$ in width. These results are consistent with previous theoretical studies [28, 29]. It is well known that the DFT-PBE method always underestimate the band gap of semiconductors, but this will not affect the following discussion on tailoring electronic properties of BNNRs through chemical decoration.

Next, chemically modified BNNRs with zigzag edges were studied. The modification was realized by decorating BNNR's edges with functional groups, such as $-\mathrm{Cl}$, $\mathrm{F},-\mathrm{NO}_{2}$, and $-\mathrm{OH}$. Here, we chose BNNR with $w=4$ as the pristine model. Both edges of the BNNR are chemically functionalized with the same or different functional groups. As shown in Figure 3, " $\mathrm{Cl}(N)-\mathrm{H}(B)$ " denotes that the BNNR is decorated with $\mathrm{Cl}$ and $\mathrm{H}$ atoms at top $(\mathrm{N})$ and bottom (B) edge, respectively.

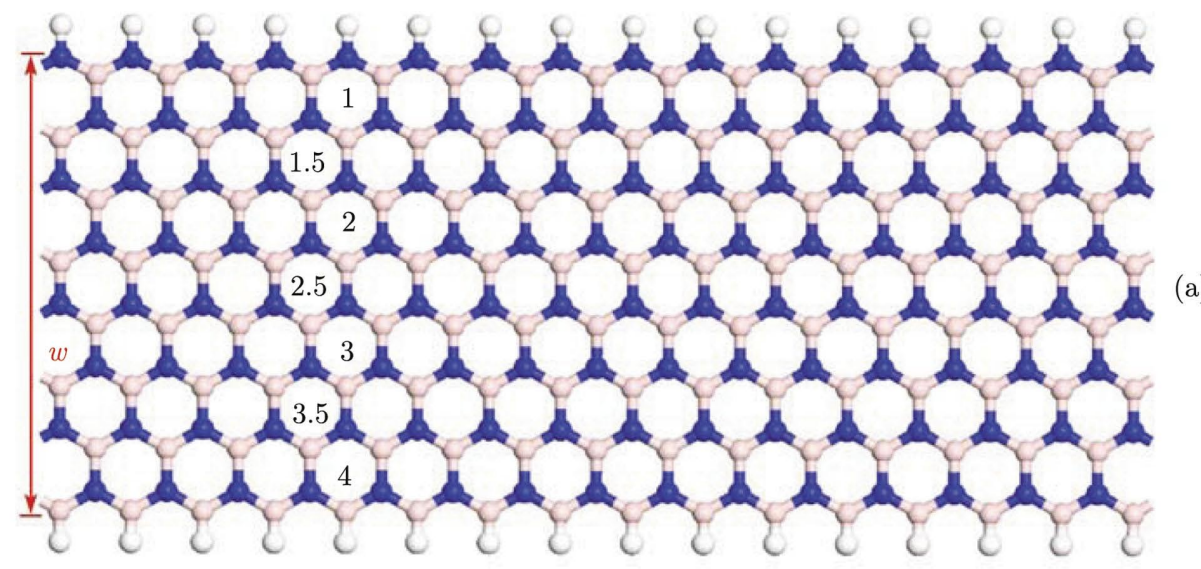

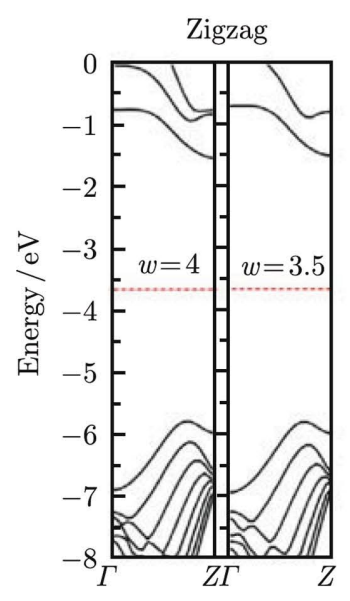

(b)

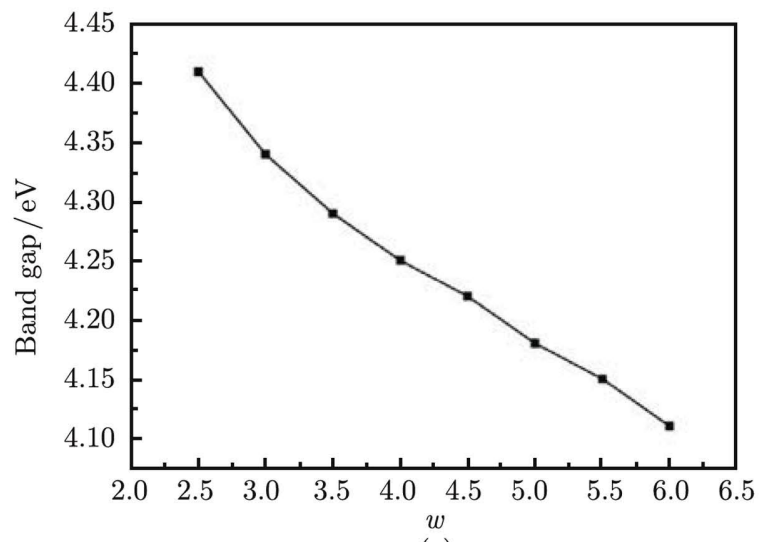

(c)

Figure 1. (a) Optimized structure of BNNR with zigzag edges. The width (w) of BNNR is 4 . (b) Calculated band structures of BNNR with $w=4$ and 3.5. (c) A relationship between band gap and $w$ is plotted. The Fermi level is plotted with a red dotted line. The blue, pink and white balls represent N, B and $\mathrm{H}$ atoms, respectively. 
Chemically DeCORATED BORON-NITRIDE NANORIBbONS

369

To assess relative stability among these chemically decorated BNNRs, we calculated average adsorption energy for various functional groups to the edges of BNNRs. The avrage adsorption energy is defined as

$$
\begin{aligned}
E= & {\left[E_{\text {total }}(\mathrm{BNNR}-\mathrm{X})+n E_{\text {total }}(\mathrm{H})\right.} \\
& \left.-E_{\text {total }}(\mathrm{BNNR}-\mathrm{H})-n E_{\text {total }}(\mathrm{X})\right] / n
\end{aligned}
$$

where $E_{\text {total }}($ system) is the total energy of the system, and $\mathrm{X}=\mathrm{F}, \mathrm{Cl}$, $\mathrm{NO}_{2}$, or $\mathrm{OH}$. Average adsorption energies are summarized in Table 1. Clearly, except $\mathrm{OH}$ and $\mathrm{F}$, the replacement reaction of $\mathrm{H}$ atom by $\mathrm{NO}_{2}$ or $\mathrm{Cl}$ is endothermic. The $\mathrm{B}$ edge of BNNR was more favorable than the $\mathrm{N}$ edge for decoration with these functional groups. The most stable configuration is the $\mathrm{H}(\mathrm{N})-\mathrm{F}(\mathrm{B})$, where the $\mathrm{B}$ edge of BNNR is saturated with $\mathrm{F}$ and the $\mathrm{N}$ edge with $\mathrm{H}$. Interestingly, the charge on functional group and corresponding $\mathrm{B}(\mathrm{N})-\mathrm{X}$ bond length are dependent on the adsorbing sites (B or $\mathrm{N}$ edge) and the type of functional groups, as shown in Table 1.

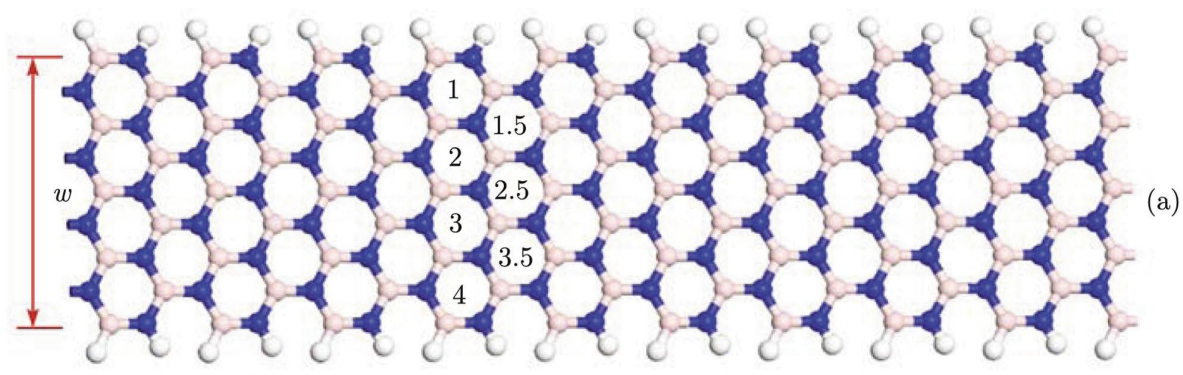

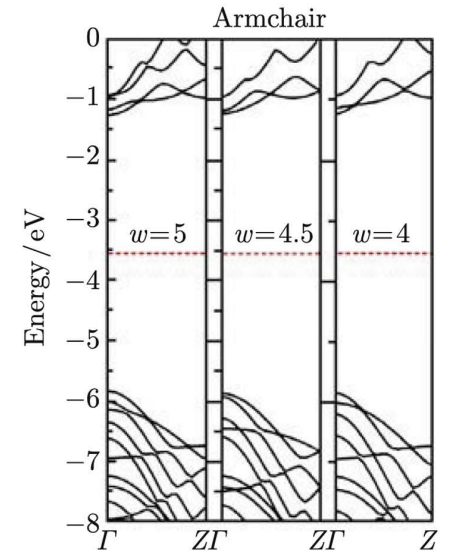

(b)

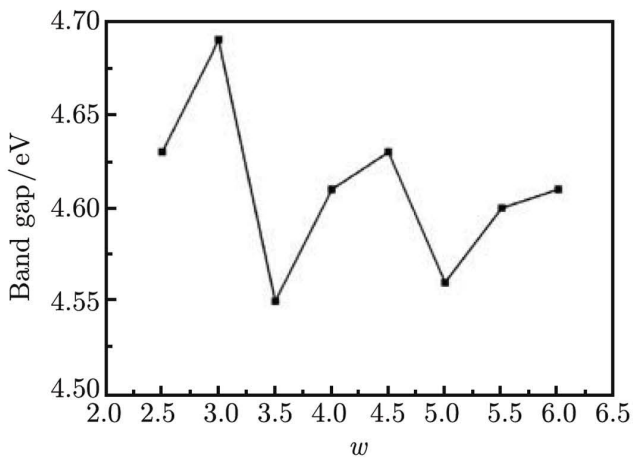

(c)

Figure 2. (a) Optimized structure of BNNR with armchair edges. The width of BNNR is $w=4$. (b) Calculated band structures of BNNRs with $w=4,4.5$ and 5. (c) A relationship between band gap and $w$. The Fermi level is plotted with a red dotted line. The blue, pink and white balls represent $\mathrm{N}, \mathrm{B}$ and $\mathrm{H}$ atoms, respectively.

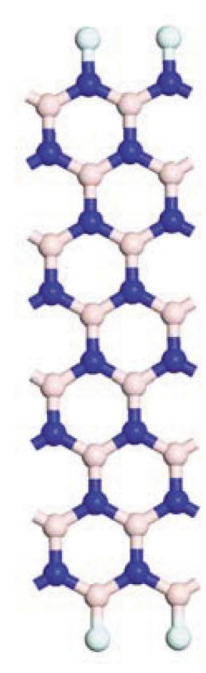

F

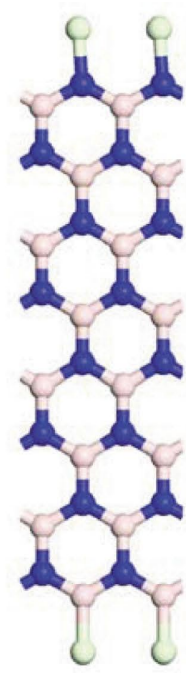

$\mathrm{Cl}$

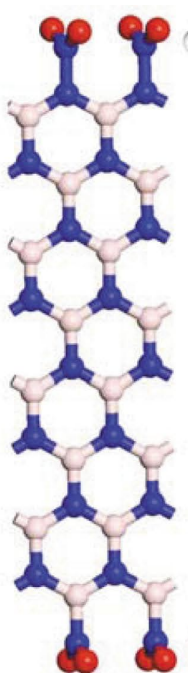

$\mathrm{NO}_{2}$

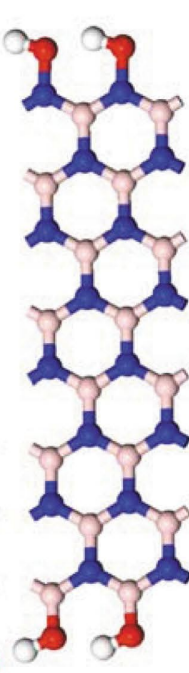

$\mathrm{OH}$

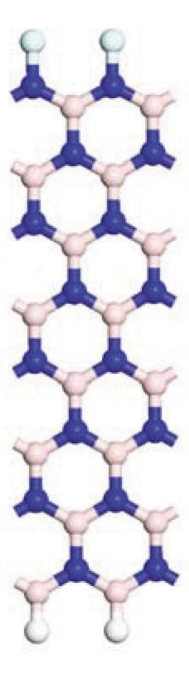

$\mathrm{Cl}_{(\mathrm{N})}-\mathrm{H}_{(\mathrm{B})}$

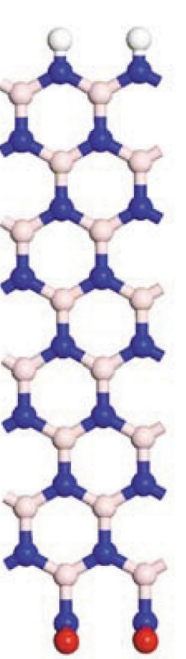

$\mathrm{H}_{(\mathrm{N})}-\mathrm{NO}_{2(\mathrm{~B})}$

Figure 3. Optimized structures of BNNRs with chemically modified zigzag edges. 
Table 1. Energy band gaps of BNNRs with chemically modified zigzag edges; adsorption energies of chemical functional group at the edge of BNNRs ( $E$ in unit of eV/group); and charge analysis results and bond lengths.

\begin{tabular}{ccccc}
\hline & E/(eV/group) & Charge $(e)$ & Gap/eV & Bond length/A \\
\hline $\mathrm{H}$ & 0.00 & $0.11^{*},-0.05^{* *}$ & 4.25 & $1.02^{\mathrm{a}}, 1.20^{\mathrm{b}}$ \\
$\mathrm{F}$ & -0.696 & $-0.06,-0.12$ & 4.51 & $1.40,1.34$ \\
$\mathrm{Cl}$ & 1.864 & $0.02,-0.06$ & 0.30 & $1.73,1.74$ \\
$\mathrm{NO}_{2}$ & 2.783 & $-0.01,-0.16$ & 1.64 & $1.50,1.52$ \\
$\mathrm{OH}$ & -0.096 & $-0.08,-0.02$ & 3.86 & $1.44,1.39$ \\
$\mathrm{H}(\mathrm{N} / \mathrm{B})-\mathrm{F}(\mathrm{B} / \mathrm{N})$ & $-2.81 / 1.42$ & $0.12,-0.12 /-0.06,-0.05$ & $4.56 / 4.32$ & $1.02,1.34 / 1.40,1.20$ \\
$\mathrm{H}(\mathrm{N} / \mathrm{B})-\mathrm{Cl}(\mathrm{B} / \mathrm{N})$ & $0.53 / 3.20$ & $0.12,-0.06 / 0.02,-0.05$ & $0.98 / 0.90$ & $1.02,1.74 / 1.73,1.20$ \\
$\mathrm{H}(\mathrm{N} / \mathrm{B})-\mathrm{NO}_{2}(\mathrm{~B} / \mathrm{N})$ & $1.91 / 3.56$ & $0.12,-0.16 /-0.02,-0.05$ & $1.86 / 2.21$ & $1.02,1.52 / 1.49,1.20$ \\
$\mathrm{H}(\mathrm{N} / \mathrm{B})-\mathrm{OH}(\mathrm{B} / \mathrm{N})$ & $-1.93 / 1.74$ & $0.11,-0.08 /-0.02,-0.05$ & $4.77 / 3.41$ & $1.02,1.39 / 1.44,1.20$ \\
$\mathrm{OH}(\mathrm{N} / \mathrm{B})-\mathrm{NO}_{2}(\mathrm{~B} / \mathrm{N})$ & $1.84 / 0.84$ & $-0.01,-0.16 /-0.02,-0.08$ & $1.08 / 2.12$ & $1.44,1.52 / 1.49,1.39$ \\
\hline
\end{tabular}

${ }^{*}$ Charge analysis result for the chemical group connected to the N-edge of BNNR.

${ }^{* *}$ Charge analysis result for the chemical group connected to the B-edge of BNNR.

${ }^{\mathrm{a}}$ and ${ }^{\mathrm{b}}$ are lengths of $\mathrm{N}-\mathrm{X}$ and $\mathrm{B}-\mathrm{X}$ bond $\left(\mathrm{X}=\mathrm{H}, \mathrm{F}, \mathrm{Cl}, \mathrm{NO}_{2}\right.$, and $\left.\mathrm{OH}\right)$, respectively.

In Figure 4, electronic band structures and density of states (DOS) projected onto functional groups are plotted. The chemical decoration of BNNR's edges may alter electronic properties of BNNRs in different fashions. For BNNRs decorated with $\mathrm{F}$ and $\mathrm{OH}$, the interaction between functional groups and $\mathrm{B}$ or $\mathrm{N}$ atoms at the edges shifts the band states near the Fermi level, resulting in an enlarged $(\mathrm{F})$ or narrowed $(\mathrm{OH})$ energy band gap. For BNNRs decorated with $\mathrm{Cl}$ and $\mathrm{NO}_{2}$, however, the functional groups induce many new states inside the band gap of pure BNNR, resulting in a very narrow band gap. These two distinct changes on the electronic properties of BNNRs offer a chemical way to control the band gap of BNNRs, as demonstrated in Table 1.

We also studied chemically modified BNNRs with armchair edges. In this case, we chose BNNRs with width $w=6$ as the pristine model. Optimized structures of BNNRs with chemically modified armchair edges are displayed in Figure 5. Unlike BNNRs with zigzag edges, the fully decorated BNNR with $\mathrm{Cl}$ is unstable and $\mathrm{Cl}$ atoms tend to escape from the edges spontaneously. A full decoration with $\mathrm{OH}$ group will induce a local distortion at the edges. The structural distortion is mainly caused by the short distance between two neighboring decorated sites. However, BNNRs with a $50 \%$ edge decoration with $\mathrm{Cl}$ or $\mathrm{OH}$ groups are very stable without showing any local structural distortion.

In Table 2, adsorption energies of various functional groups to BNNR's edges are summarized. Similar to BNNRs with zigzag edges, $B$ edges are more favorable than $\mathrm{N}$ edges for the adsorption. Moreover, the BNNR with Bedge-site decoration ( $50 \%$ decoration) is energetically more stable than pure BNNR, suggesting that the decoration may be realized experimentally.

In Figure 6, we plotted electronic band structures and DOS of BNNRs with chemically modified armchair edges. The interaction between functional groups and BNNR mainly causes a shift of band states in most cases. An exception is that when only N-edge-site is decorated (50\% decoration) with $\mathrm{Cl}$, an unoccupied impurity state is introduced inside the band gap due to the interaction between $\mathrm{Cl}$ and the $\mathrm{N}$ edge. As listed in Table 2, most BNNRs with chemi- cally modified armchair edges have a narrowed band gap, except " $\mathrm{H}(\mathrm{N})-\mathrm{OH}(\mathrm{B})$ " and " $\mathrm{H}(\mathrm{B})-\mathrm{F}(\mathrm{N})$ " decorated BNNRs which have a larger band gap than the pure BNNR.
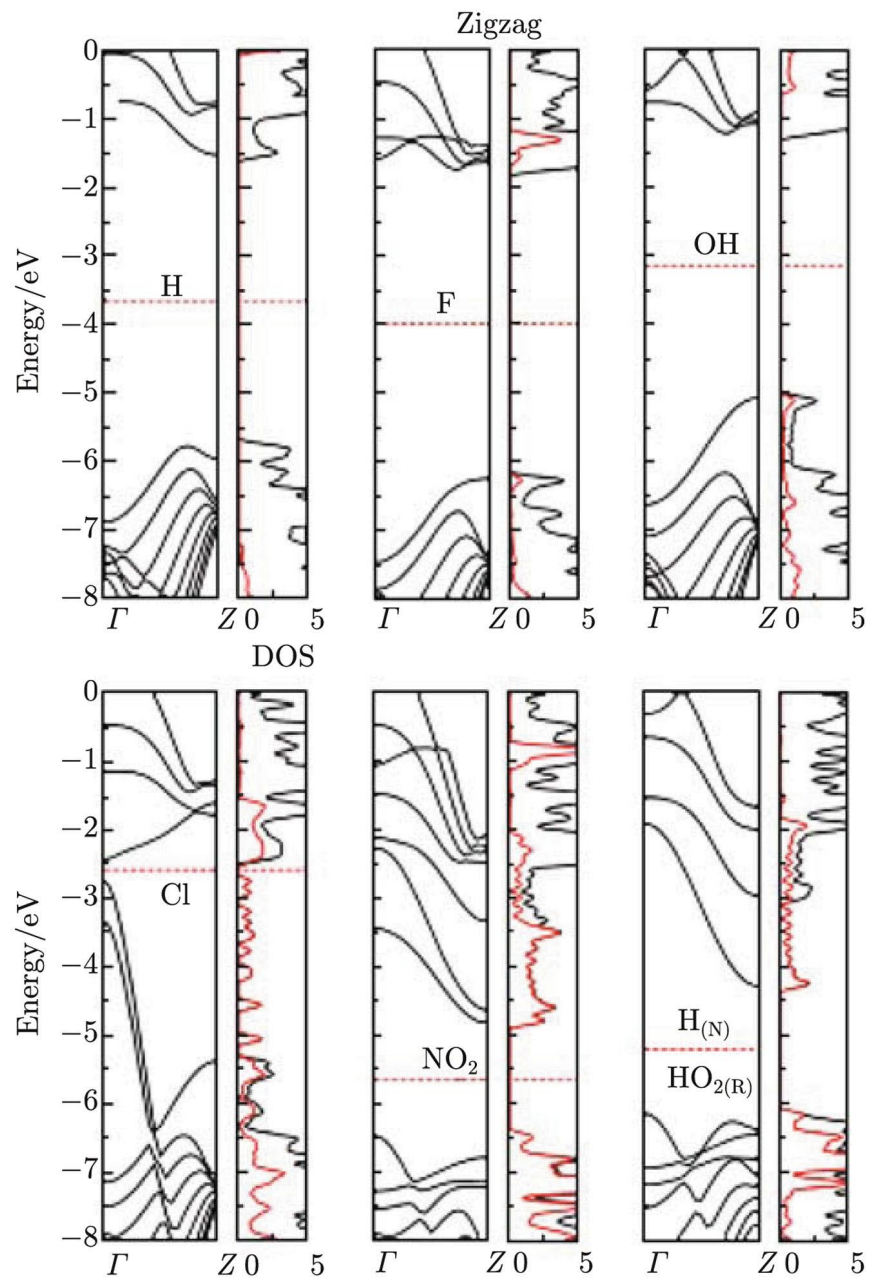

Figure 4. Electronic band structures and density of states (DOS) of BNNRs with chemically modified zigzag edges. The Fermi level is plotted with a red dashed line. The DOS projected onto functional groups are plotted with red solid lines. 
Table 2. Energy band gaps of BNNRs with chemically modified armchair edges; the adsorption energies of chemical functional group to BNNR ( $E$ in unit of $\mathrm{eV} /$ group); and charge analysis results and bond lengths.

\begin{tabular}{ccccc}
\hline & $E /(\mathrm{eV} /$ group $)$ & Charge $(e)$ & $\mathrm{Gap} / \mathrm{eV}$ & Bond/A \\
\hline $\mathrm{H}$ & 0.00 & $0.11^{*},-0.06^{* *}$ & 4.61 & $1.02^{\mathrm{a}}, 1.21^{\mathrm{b}}$ \\
$\mathrm{F}$ & -0.30 & $-0.05,-0.12$ & 4.48 & $1.39,1.33$ \\
$\mathrm{OH}$ & 0.19 & $0.04,0.13$ & 4.31 & 1.38 \\
$\mathrm{H}(\mathrm{N} / \mathrm{B})-\mathrm{F}(\mathrm{B} / \mathrm{N})$ & $-2.90 / 1.59$ & $0.10,-0.12 /-0.04,-0.05$ & $4.48 / 4.73$ & $1.02,1.37 / 1.42,1.18$ \\
$\mathrm{H}(\mathrm{N} / \mathrm{B})-\mathrm{Cl}(\mathrm{B} / \mathrm{N})$ & $-0.57 / 2.77$ & $0.06,-0.04 / 0.07,-0.08$ & $4.57 / 3.58$ & $1.01,1.81 / 1.76,1.16$ \\
$\mathrm{H}(\mathrm{N} / \mathrm{B})-\mathrm{OH}(\mathrm{B} / \mathrm{N})$ & $-1.24 / 2.21$ & $0.09,-0.07 / 0.01,-0.05$ & $4.79 / 3.87$ & $1.01,1.40 / 1.43,1.19$ \\
\hline
\end{tabular}

${ }^{*}$ Charge analysis result for the chemical group decorating the N-edge of BNNR.

${ }^{* *}$ Charge analysis result for the chemical group decorating the B-edge of BNNR.

${ }^{\mathrm{a}}$ and ${ }^{\mathrm{b}}$ are length of $\mathrm{N}-\mathrm{X}$ and $\mathrm{B}-\mathrm{X}$ bonds $(\mathrm{X}=\mathrm{H}, \mathrm{F}, \mathrm{Cl}$, and $\mathrm{OH})$, respectively.

Figure 5. Optimized structures of BNNRs with chemically modified armchair edges.

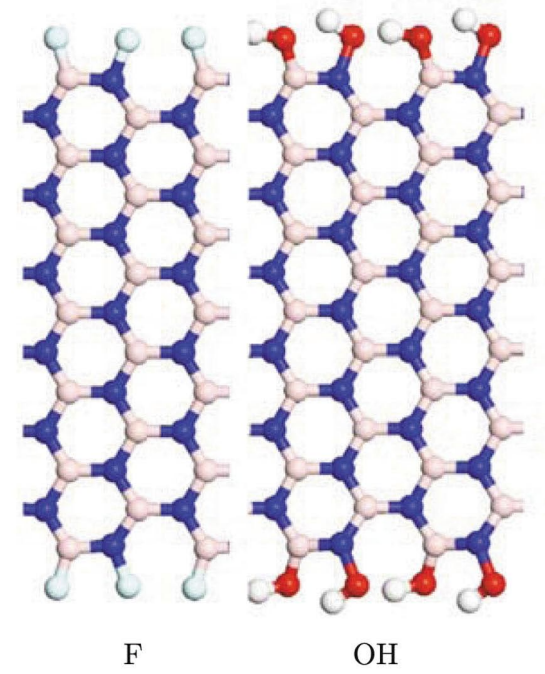

$\mathrm{OH}$
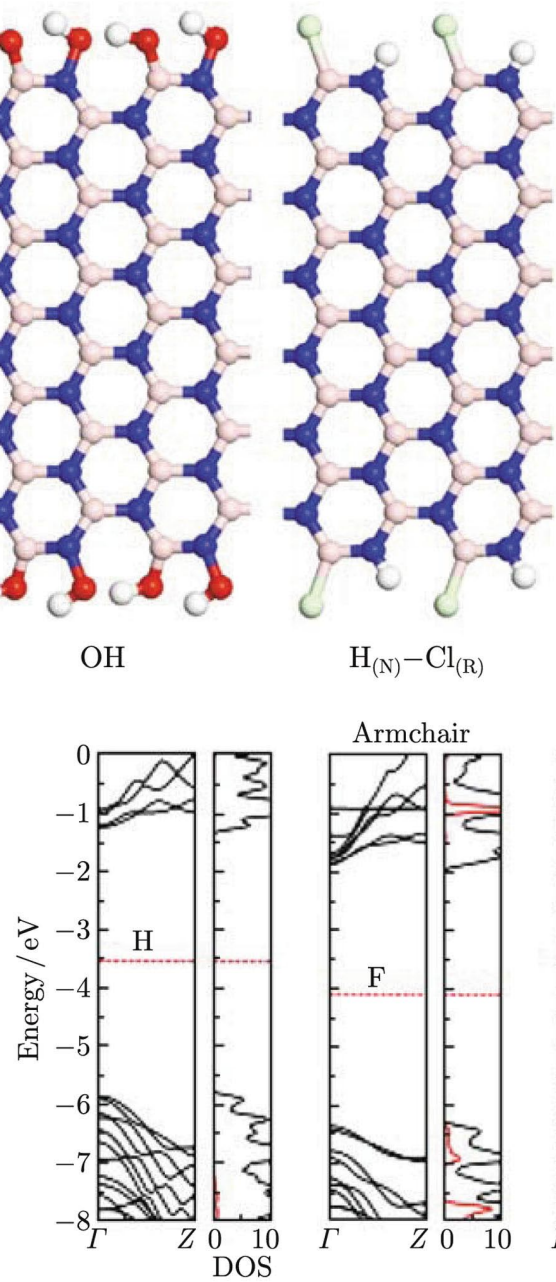

$\mathrm{H}_{(\mathrm{N})}-\mathrm{Cl}_{(\mathrm{R})}$

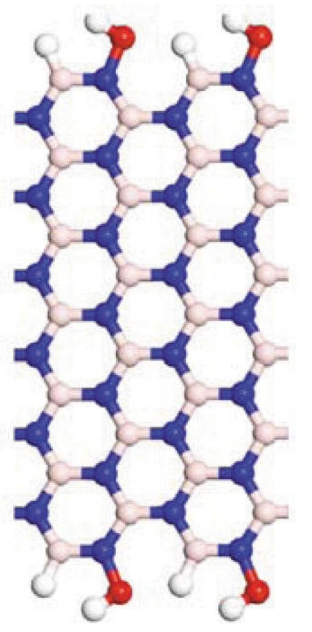

$\mathrm{H}_{(\mathrm{R})}-\mathrm{OH}_{(\mathrm{N})}$
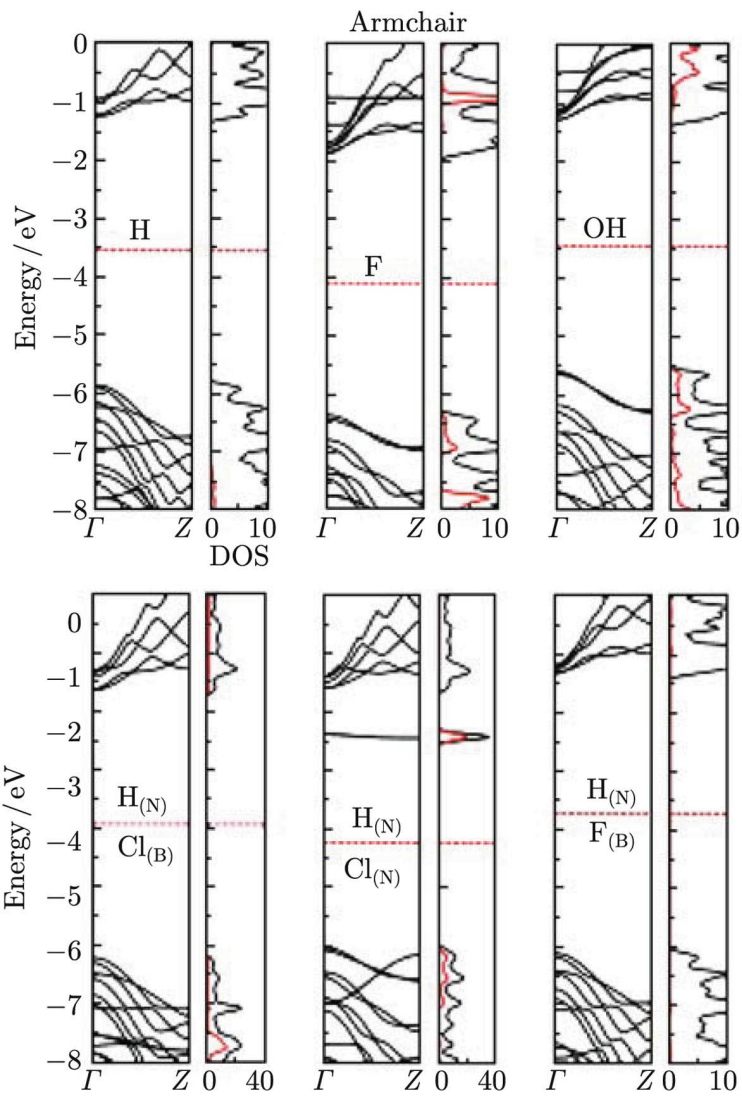

\section{Acknowledgments}

This work was supported by grants from the National Science Foundation (Grant Nos. CHE-0427746, CHE- 0701540 and CMMI-0709333), the Nebraska Research Initiative, the National Science Foundation of China (Grant No. 20628304), and by the Research Computing Facility at University of Nebraska-Lincoln and Holland Supercomputing Center at University of Nebraska-Omaha.
Figure 6. Electronic band structures and density of states (DOS) NRs with chemically modified armchair edges. The Fermi level is plotted with a red dashed line. The DOS projected onto functional groups are plotted with red solid lines.

properties, particularly its band gap. The band-gap modulation by chemical decoration may be exploited for nanoelectronic applications.

conclusion, we have studied structural stability and NRs using a density functional theory method. Electronic properties of pure BNNRs depend on the type of edges edges are semiconducting with wide band gaps. The band gap of BNNRs with zigzag edges decreases monotonically edges oscillates periodically with the width. Chemical decoration of BNNRs' edges can modify BNNRs' electronic 


\section{References}

1. K. S. Novoselov, A. K. Geim, S. V. Morozov, D. Jiang, Y. Zhang, S. V. Dubonos, I. V. Grigorieva, and A. A. Filrsov, Science, 2004, 306: 666

2. K. S. Novoselov, A. K. Geim, S. V. Morozov, D. Jiang, M. I. Katsnelson, I. V. Grigorieva, S. V. Dubonos, and A. A. Firsov, Nature, 2005, 438: 197

3. Y. B. Zhang, Y. W. Tan, H. L. Stormer, and P. Kim, Nature, 2005, 438: 201

4. C. Berger, Z. M. Song, X. B. Li, X. S. Wu, N. Brown, C. Naud, D. Mayou, t. B. Li, J. Hass, A. N. Marchenkov, E. H. Conrad, P. N. First, and W. A. de Heer, Science, 2006, 312: 1191

5. C. Berger, Z. M. Song, T. B. Li, X. B. Li, A. Y. Ogbazghi, R. Feng, Z. T. Dai, A. N. Marchenkov, E. H. Conrad, P. N. First, and W. A. de Heer, J. Phys. Chem. B, 2004, 108: 19912

6. M. Y. Han, B. “ Ozyilmaz, Y. B. Zhang, and P. Kim, Phys. Rev. Lett., 2007, 98: 206805

7. X. L. Li, X. R. Wang, L. Zhang, S. Lee, and H. J. Dai, Science, 2008, 319: 1229

8. K. Nakada and M. Fujita, Phys. Rev. B, 1996, 54: 17954

9. Y. Miyamoto, Phys. Rev. B, 1999, 59: 9858

10. H. Lee, Y. W. Son, N. Park, S. Han, and J. Yu, Phys. Rev. B, 2005, 72: 174431

11. M. Ezawa, Phys. Rev. B, 2006, 73: 045432

12. Y. W. Son, M. L. Cohen, and S. G. Louie, Phys. Rev. Lett., 2006, 97: 216803

13. V. Barone, O. Hod, and G. E. Scuseria, Nano Lett., 2006, 6: 2748

14. Q. M. Yan, B. Huang, J. Yu, F. W. Zheng, J. Zang, J. Wu, B. L. Gu, F. Liu, and W. H. Duan, Nano Lett., 2007, 7: 1469

15. Z. F. Wang, Q. W. Shi, Q. X. Li, X. P. Wang, J. G. Hou, H. X. Zheng, Y, Yao, and J. Chen, App. Phys. Lett., 2007, 91: 053109

16. X. J. Wu and X. C. Zeng, Nano Res., 2008, 1: 40
17. W. L. Wang, S. Meng, and E. Kaxiras, Nano Lett., 2008, 8: 241

18. J. Fernndez-Rossier and J. J. Palacios, Phys. Rev. Lett., 2007, 99: 177204

19. O. Hod, J. E. Peralta, and G. E. Scuseria, Phys. Rev. B, 2007, 76: 233401

20. O. Hod, V. Barone, and G. E. Scuseria, Phys. Rev. B, 2008, 77: 035411

21. M. Ezawa, Phys. Rev. B, 2007, 76: 245415

22. M. Ezawa, Physica E, 2008, 40: 1421

23. Y. W. Son, M. L. Cohen, and S. G. Louie, Nature, 2006, 444: 347

24. E. Rudberg, P. Satek, and Y. Luo, Nano Lett., 2007, 7: 2211

25. O. Hod, V. Barone, J. E. Peralta, and G. E. Scuseria, Nano Lett., 2007, 7: 2295

26. E. J. Kan, Z. Y. Li, J. L. Yang, and J. G. Hou, J. Am. Chem. Soc., 2008, 130: 4224

27. K. S. Novoselov, A. K.Geim, and S. V. Morozov, Proc. Natl. Acad. Sci. USA, 2005, 102: 10451

28. A. J. Du, S. C. Smith, and G. Q. Lu, Chem. Phys. Lett., 2007, 477: 181

29. Z. H. Zhang and W. L. Guo, Phys. Rev. B, 2008, 77: 075403

30. X. F. Gao, Z. Zhou, Y. L. Zhao, S. Nagase, S. B. Zhang, and Z. F. Chen, J. Phys. Chem. C, 2008, 112: 12677

31. F. W. Zheng, Z. R. Liu, J. Wu, W. H. Duan, and B. L. Gu, Phys. Rev. B, 2008, 78: 085423

32. C. H. Park and S. G. Louie, Nano Lett., 2008, 8: 2200

33. V. Barone and J. E. Peralta, Nano Lett., 2008, 8: 2210

34. F. W. Zheng, G. Zhou, Z. R. Liu, J. Wu, W. H. Duan, B. L. Gu, and S. B. Zhang, Phys. Rev. B, 2008, 78: 205415

35. B. Delley, J. Chem. Phys., 1990, 92: 508

36. B. Delley, J. Chem. Phys., 2003, 113: 7756

37. J. P. Perdew, K. Burke, and M. Ernzerhof, Phys. Rev. Lett., 1996, 77: 3865

38. H. J. Monkhorst and J. D. Pack, Phys. Rev. B, 1976, 13: 5188 